\section{Como Monitorar o Impacto dos Visitantes Utilizando o Sistema LAC - Limite Aceitável de Câmbio}

How to Monitor Visitor Impact Using the LAC (Limits of Acceptable Change) System

\section{Leide Yassuco Takahashi Antonio Cristiano Vieira Cegana ${ }^{2}$}

RESUMO: Este levantamento tem por objetivo discutir os principais sistemas de manejo dos impactos dos visitantes nas unidades de conservação. A busca por atividades recreativas em áreas naturais com características ímpares tem crescido a cada ano, e o grande desafio de seus administradores é como fazer para minimizar os impactos gerados pelo uso público. A escassez de recursos humanos adequados, a falta de literatura, de pesquisas e de divulgação dos resultados sobre a questão limitam a utilização dos sistemas no Brasil. Dessa forma, é essencial promover uma discussão clara sobre os aspectos limitantes e os aspectos positivos dos sistemas, destacando as pesquisas e recomendações mais recentes.

PALAVRAS-CHAVE: unidades de conservação; recreação; indicadores de impacto.

ABSTRACT: The goal of this study is to discuss the main visitor impact management system in protected areas. The demand for recreational activities in natural areas with unique characteristics has increased in the last years and the manager's challenge is how to reduce the impacts of public use. The

1. Doutora em Conservação da Natureza; mestre em Ciências Florestais; bacharel em Engenharia Florestal pela Universidade Federal do Paraná. Professora adjunta do Departamento de Agronomia da Universidade Estadual de Maringá - PR. Contato: Av. Colombo, 5.790 - 87020-900 - Maringá-PR; e-mail: ladi@uem.br.

2. Mestrando em Agronomia pela Universidade Estadual de Maringá - PR; bacharel em Agronomia pela mesma instituição. Consultor em manejo do uso público em áreas naturais. Contato: Rua Neo Alves Martins, 1.363/101, Zona 3-87050-110 - Maringá-PR; e-mail: agrocris@pop.com.br. personnel scarcity, the lack of specific literature, research and diffusion of the results about this issue, restrain the application of these systems in Brazil. Therefore, is essential to promote a wide discussion about positive and negative aspects of each system, emphasizing recent researches and specialist board recommendation.

KEYWORDS: protected areas; recreation; impact indicators.

\section{Introdução}

Os estudos para avaliação dos impactos causados pelos visitantes em unidades de conservação começaram a ser desenvolvidos no Brasil na década de 1990, e pode-se dizer que avançaram bastante nos últimos anos. A vasta literatura estrangeira, em parte disponível na internet, tem contribuído para divulgar novas propostas e, de certa forma, estimular novas pesquisas.

As metodologias utilizadas fora do Brasil, adaptadas à nossa realidade, têm trazido à tona diferentes preocupaçōes em relação ao planejamento e manejo do uso público dentro das unidades de conservação. Isso fez despertar um maior interesse pelos sistemas de planejamento e manejo do impacto dos visitantes, com o objetivo de avaliar, monitorar e minimizar os danos.

De modo geral, os impactos gerados pela participação nessas atividades em áreas naturais decorrem mais da falta de um planejamento do que do mau uso propriamente dito. Essa situação se agrava em decorrência da limitação de recursos financeiros e humanos para a fiscalização e proteção das áreas.

Como o Brasil é um país com vasta extensão territorial e poucas unidades de conservação (segundo dados estatísticos apresentados pelo IBAMA, até 28/08/ 2003 as áreas protegidas totalizavam 53.806 .450 hectares $-6,3 \%$ da superfície do país), não há motivos para oferecer inúmeras opçōes recreativas em todas as áreas. As unidades de proteção integral (23.761.582 hectares - 2,78\% do território brasileiro) existem em menor quantidade se comparadas às de uso sustentado (30.044.868 hectares - 3,52\% do território). Assim, é essencial considerar as limitaçōes anteriormente apresentadas antes de se pensar em promover um amplo uso recreativo desses locais.

Embora algumas pesquisas revelem que a maioria dos visitantes não conhece regras de mínimo impacto, não há dúvidas de que é preciso divulgar melhor a importância das unidades de conservação e o comportamento esperado por parte desses visitantes. Além disso, a necessidade de atender à demanda, em termos de disponibilizar atividades recreativas, só é válida e justificável quando essa busca não fere os princípios da conservação dos recursos naturais. 
Diante deste quadro, considerando que grande parte dos atrativos naturais está localizada dentro das unidades de conservação e que a demanda aumenta a cada ano, o grande desafio dos administradores das áreas naturais protegidas consiste em compatibilizar as atividades recreativas, respeitando os princípios de criação da área.

O sistema Limits of Acceptable Change - LAC aqui apresentado tem o objetivo de discutir um dos sistemas de planejamento mais utilizados pelos norte-americanos, e de destacar algumas experiências realizadas no Brasil.

\section{Histórico}

Segundo Hendee \& Dawson (2002), a capacidade de carga é um conceito fundamental no manejo dos recursos e do ambiente natural, podendo ser definido como o nível máximo de uso que uma área pode tolerar, considerando os fatores do meio.

O desenvolvimento formal da idéia de capacidade de carga recreativa começou com a monografia publicada por Alan Wagar, em 1964. Na verdade, não se tratava de um novo conceito, mas de uma reformulação do conceito de capacidade de carga animal (Cole \& Stankey, 1997). Em razão da origem do conceito, os administradores das áreas protegidas compararam incorretamente a capacidade de carga recreativa, apenas com a determinação do número de pessoas que poderiam visitar uma área sem provocar danos ao ecossistema. A aplicação desta metodologia em vários locais nos Estados Unidos da América deveu-se ao fato de que, em 1978, o decreto 95-625 exigia que cada Parque Nacional norte-americano desenvolvesse estudos de capacidade de carga das áreas de uso público (McCool, 1996).

Para Stankey et al. (1985), a comprovação de que não existe relação direta entre número de visitantes e quantidade de impactos gerados, e de que estes estão muito mais ligados ao comportamento dos visitantes do que ao número de pessoas, confirmou o quanto essa metodologia não era adequada. Mais importante do que o fato de a complexidade da capacidade de carga recreativa não ter sido bem entendida, é o fato de ela implicar uma relação causa-efeito entre a quantidade de uso e os impactos gerados numa área. Monitorar somente os impactos sobre os recursos biofísicos não contribui para estabelecer a capacidade-limite (Hammitt \& Cole, 1998).

Em essência, essa metodologia deixou de ser utilizada porque enfatizava muito a questão “Quantas pessoas são demais naquela área?”, quando a questão principal para os administradores deveria ser "Quais são as condições adequadas ou aceitáveis de visitação e como podemos alcançá-las?” (Borrie, McCool \& Stankey, 1998).
A primeira articulação do conceito LAC surgiu em 1963, num estudo sobre os impactos do acampamento em uma área protegida norte-americana. Frissel concluiu que, se o uso recreativo é permitido, o impacto do uso no local do acampamento é inevitável e deve ser aceito. Entretanto, esse autor afirma que um limite deveria ser estabelecido na quantidade de mudança a ser tolerada (Hammitt \& Cole, 1998).

Reconhecendo que o objetivo da capacidade da carga recreativa era a busca pelo equilíbrio entre o uso e a proteção dos recursos, Frissell \& Stankey (1972) propuseram o conceito de LAC como um modelo para tomada de decisão sobre capacidade de carga. A idéia fundamental era destacar a importância do manejo dos visitantes para alcançar os objetivos específicos da área (Hammitt \& Cole, 1998).

Após esta proposição, McCool (1996), Borrie, McCool \& Stankey (1998) destacaram que vários outros processos de planejamento do uso público foram desenvolvidos, tais como Washburne's Alternative Carrying Capacity Model; Carrying Capacity Assessment Process - C-Cap; Visitor Activity Management Process - VAMP, usado nos parques do Canadá; Visitor Impact Management - VIM; e, mais recentemente, o Visitors Experience and Resource Protection - VERP.

Segundo Hammitt \& Cole (1998), os processos LAC, VIM e VERP utilizam terminologias e seqüência de etapas sutilmente diferentes. Entretanto, os participantes do workshop sobre o LAC, realizado na Universidade de Montana, EUA, em maio de 1997, concordaram que esses processos foram conceitualmente idênticos, e que a seqüência de etapas não precisa ser rigorosamente seguida por se tratar de um procedimento amplamente interativo.

Desde a proposição do LAC, em 1985, tem-se observado um efeito positivo de sua aplicação, tanto nos EUA como em outros países. Conforme Hendee \& Dawson (2002), esse conceito vem sendo gradualmente aperfeiçoado, tornandose uma ferramenta útil no planejamento e manejo de áreas naturais, principalmente onde existem conflitos de uso, substituição de objetivos e questões sobre o controle de mudanças inaceitáveis.

\section{Importância dos sistemas de planejamento e manejo do uso público em áreas naturais}

Após a Segunda Guerra Mundial, os países desenvolvidos passaram por uma rápida industrialização e conseqüente aumento do tempo disponível. Com isso, o crescimento da população e sua concentração nos centros urbanos, a melhoria das estradas e aeroportos, a melhoria dos meios de comunicação e do padrão de vida e, essencialmente, o desenvolvimento e popularização do automóvel contri- 
buíram para o fortalecimento da recreação ao ar livre. Como a visitação em áreas naturais cresceu sem precedentes a partir dessa época, o maior desafio dos administradores e pesquisadores, no final da década de 1970, era como atender às necessidades da demanda sem degradar o ambiente.

A compreensão das implicações do uso público em áreas naturais é de essencial importância para o manejo delas. Em relação à fauna, Fennell (2002) destaca que o fator humano, a manipulação e a exploração dos recursos naturais são os pontos que mais prejudicam o equilíbrio dentro e entre as espécies.

As restrições de manejo, tais como a limitação de recursos, tanto em termos de pessoal quanto em termos de tempo, devem ser consideradas cuidadosamente na seleção do sistema de planejamento e gestão de uma área. Como o controle dos impactos da recreação afeta a qualidade dos recursos recreativos e a experiência dos visitantes, muitas vezes as decisões são polêmicas. Por exemplo: restringir o uso do acampamento pode reduzir o impacto, mas impõe um controle na liberdade de viajar e escolher a área de acampamento desejada (Leung \& Marion, 2000).

Segundo Leung \& Marion (2000), os sistemas de planejamento para o manejo do uso público mais utilizados nos EuA são o LAC e o VERP. No Brasil, embora poucas áreas tenham recebido algum planejamento em termos de uso público, as propostas mais comumente efetuadas se referem à capacidade de carga (Cifuentes, 1992), LAC (Stankey et al., 1985) e VIM (Graefe, Kuss \& Vaske, 1990).

A capacidade de carga proposta por Cifuentes foi adotada no Brasil, por alguns técnicos, como modelo para definir a quantidade ideal de pessoas numa área, mas foi gradualmente substituído por sistemas mais completos. Vale ressaltar que, em 1996, participando de um grupo de pesquisa no Parque Nacional de Galápagos, Cifuentes introduziu na proposta de capacidade de carga tradicional duas das três principais etapas presentes no LAC e no VIM, quais sejam: a definição de indicadores de impactos e o monitoramento desses indicadores.

No Brasil, o LAC e o vim começaram a ser estudados mais profundamente por pesquisadores de instituições de ensino e pesquisa em meados da década de 1990, e vêm estimulando outros interessados a trabalhar com o tema em diferentes áreas.

O LAC é um sistema específico para mensurar os impactos da visitação que avalia se os objetivos de redução ou mitigação de impactos estão ou não sendo atingidos (Drumm \& Moore, 2003). Como qualquer tipo de uso produz algum impacto, as mudanças ocorridas são inevitáveis. Desta forma, procura-se definir quanto de mudança será permitido e quais ações de manejo serão necessárias para controlá-las (Takahashi, 1997). Embora esse sistema tenha sido desenvolvido para avaliar impactos da atividade recreativa, nos últimos anos ele vem sendo utilizado amplamente para outras questões de manejo por tratar-se de um conceito mais qualitativo do que quantitativo (Strasdas, 2002).
Considerando que os sistemas LAC, VIM e VERP utilizam uma seqüência de etapas semelhantes e não apresentam diferenças significativas, os especialistas do workshop Limits of acceptable change and related planning processes: progress and future directions, realizado em Montana, EUA, em 1997, ressaltam que os esforços devem ser envidados no que tange à avaliação dos melhores indicadores e não à discussão dos sistemas.

\section{Sistema de planejamento LAC}

O LAC proporciona um marco sistemático de tomada de decisão que ajuda a determinar quais recursos e quais condições recreativas são aceitáveis. Essa metodologia atenua a gravidade dos conflitosproduzidos entre o tempolivre, a visitação e a conservação do ambiente, além de definir os impactos que se associam com distintos níveis de proteção ambiental (Wearing \& Neil, 1999). De acordo com Stankey et al. (1985), esse sistema é baseado em quatro elementos principais: 1) especificação das condições aceitáveis e realizáveis, definidas por vários parâmetros mensuráveis; 2) análise da relação entre as condições existentes e aceitáveis; 3) identificação das melhores ações de manejo; e 4) programa de monitoramento e efetividade do manejo da área.

De acordo com Cole \& McCool (1997), o LAC foi desenvolvido inicialmente em nove etapas distintas. Entretanto, após o workshop citado anteriormente, os especialistas recomendam a inclusão de mais uma etapa (Etapa 1) envolvendo mais explicitamente a definição de objetivos e condições desejadas. Destaca-se que a seqüência de etapas não precisa ser rigorosamente observada, uma vez que esses processos são amplamente interativos e circulares ao invés de lineares. Apresentam-se, a seguir, cada uma das etapas que compõem o LAC. A Figura 1 apresenta os principais elementos do sistema de planejamento em discussão.

\section{Etapa 1 - Definição dos objetivos e das condições desejadas}

Esta etapa envolve a reunião dos mandatos legais e políticos que guiarão o manejo das unidades, desenvolvendo uma perspectiva sobre a importância da área, suas características ímpares e sua abrangência regional e nacional. Esses mandatos podem ser usados para descrever os objetivos gerais da área e constituem o estabelecimento das condições desejadas. 


\section{Etapa 2 - Identificação dos valores e das limitações da área}

Nesta etapa inicial, deve-se identificar as características ou valores especiais que precisam ser mantidos ou alcançados; caracterizar os problemas ou interesses de manejo relevantes; e levantar as questões de âmbito econômico, social e ambiental ligadas ao público e o papel da área no contexto regional e nacional. A troca de informações entre os administradores, pesquisadores e o público é de suma importância para o entendimento e sucesso desta etapa, por se tratar da base para uma melhor compreensão dos recursos naturais, de como eles podem e devem ser manejados, focalizando as principais questões de manejo (Eagles, McCool \& Haynes, 2002).

\section{Etapa 3 - Estabelecimento do zoneamento da área}

Com base na etapa anterior, subdivide-se a área em diferentes zonas, identificando as condições biofísicas, recreativas e o tipo de manejo desejável e adequado, estabelecendo metas e normas compatíveis com os objetivos e legislação incidente em cada zona. Com essa gama de dados mapeados pode-se identificar quais tipos de informações ainda devem ser buscados, que limites aceitáveis de impacto precisam ser estabelecidos e que ações de manejo são necessárias para cada ambiente (Hendee \& Dawson, 2002).

\section{Etapa 4 - Seleção dos indicadores de impacto}

Esta etapa é um dos pontos-chave do sistema de planejamento LAC, em que são selecionadas variáveis mensuráveis que indicam o estado de conservação de cada zona. Essas variáveis podem ser de âmbito biológico, físico ou recreativo, mas que representem as condições julgadas adequadas e aceitáveis para cada área específica. A inviabilidade de avaliar todos os indicadores ecológicos e recreativos de um local obriga o estabelecimento de um grupo de indicadores que sejam eficientemente representativos e indiquem, quando necessário, as condições ambientais, econômicas, políticas e recreativas de uma área. Após a seleção dos indicadores, deve ser discriminado como e com que freqüência essas informações serão coletadas, para que seja identificado (ou para que se verifique) se as condições desejadas estão sendo mantidas ou alcançadas. Mais detalhes sobre esse tema (ou sobre esta etapa) serão encontrados no item "Seleção de indicadores de impacto", a seguir.

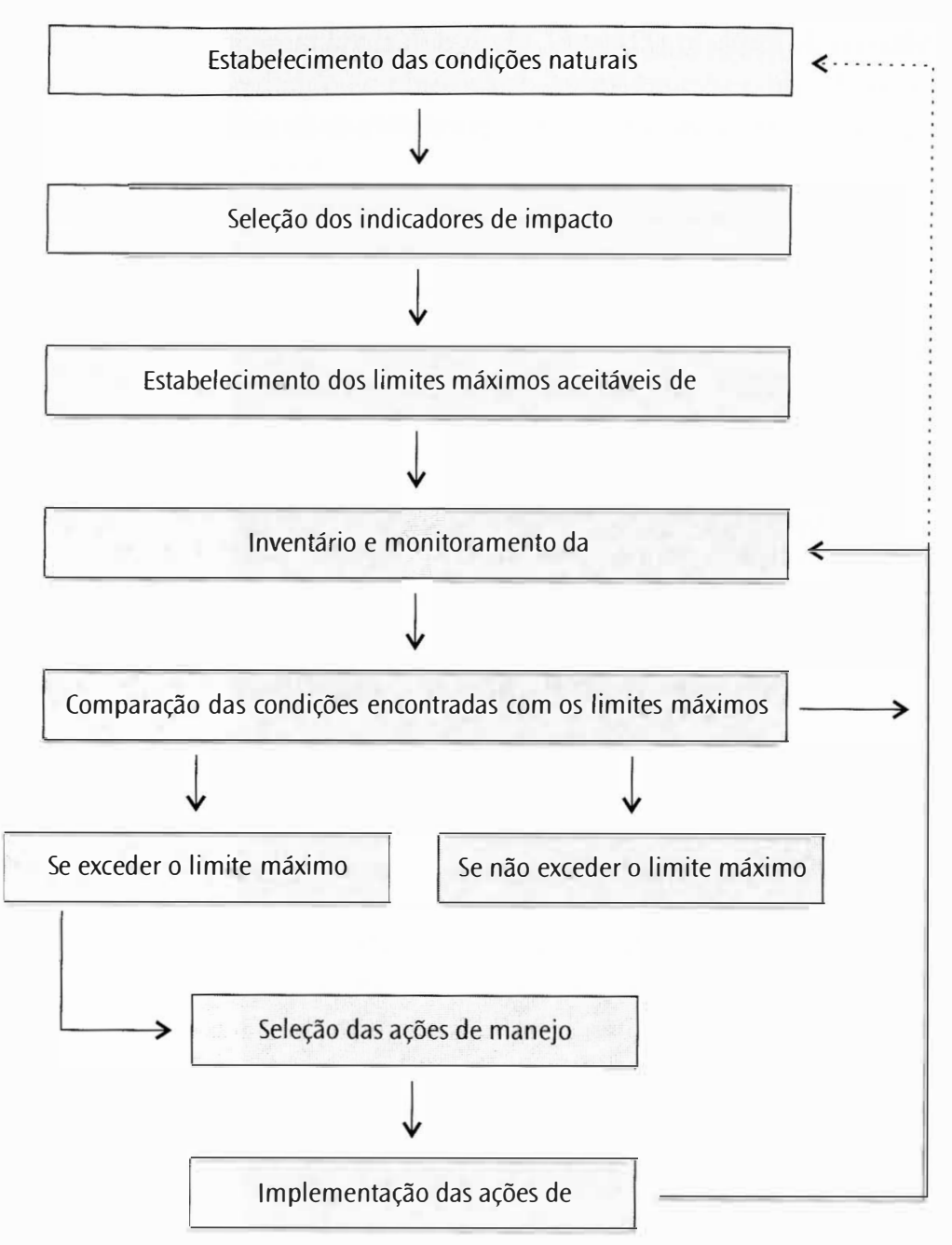

Figura 1. Principais elementos do sistema de planejamento Limits of Acceptable Change, resumido por Hendee \& Dawson (2002)

\section{Etapa 5 - Inventário das condições existentes}

Com o inventário das condições existentes obtém-se o estado atual da área, de acordo com cada indicador proposto anteriormente, evitando um levantamento de dados desnecessário. De acordo com o nível de impacto apresentado, identificase onde e quais ações de manejo devem ser tomadas para o restabelecimento das condições naturais adequadas para cada zona. $O$ inventário das condições existentes de cada zona é imprescindível para que se possam estabelecer os limites máxi- 
mos aceitáveis de impacto (Etapa 6). O nível de detalhamento desse inventário está diretamente ligado a vários fatores, destacando os objetivos da área, as condições existentes, os fatores econômicos e a disponibilidade de mão-de-obra.

\section{Etapa 6 - Estabelecimento dos limites aceitáveis de impacto}

Com as informações coletadas nas etapas anteriores, pode-se estabelecer o limite aceitável de impacto para cada indicador nas diferentes zonas. Hendee \& Dawson (2002) relatam que esses limites podem ser definidos e centrados em três metas distintas: 1) manter o nível mais elevado de conservação; 2 ) regenerar áreas já degradadas; e 3) manter ou alcançar os níveis aceitáveis de impacto. Essa fase exige, além da experiência do administrador e seu conhecimento da área, um nível técnico avançado para que os limites propostos sejam realistas e atingíveis. A cautela é imprescindível, pois um erro nesse ponto poderá acarretar impactos até mesmo irreversíveis. O item "Definição do limite aceitável de impacto", a seguir, tratará das principais características para se identificar limites aceitáveis de impacto ideais.

\section{Etapa 7 - Identificação das condições naturais e recreativas adequadas}

\section{para cada zona}

De acordo com as informações obtidas a partir dos administradores, dos visitantes e dos dados resultantes das etapas 2 e 5, define-se quais e como as condições naturais e recreativas deverão ser mantidas ou alcançadas nas diferentes zonas. Somente dessa forma as necessidades, interesses e valores da área irão prevalecer.

\section{Etapa 8 - Estabelecimento de ações de manejo para cada condição}

Ao comparar as condições existentes na Etapa 5 com os limites máximos aceitáveis de impacto da Etapa 6, pode-se detectar se um determinado local apresenta ou não problemas. Dessa forma, esta fase é responsável pela identificação de potenciais ações de manejo que possam reverter a situação de cada indicador selecionado e implantado, de acordo com os recursos econômicos e sociais da área em questão. Como relata Kuss, Graefe \& Vaske (1990), essas ações podem ser indiretas, com implantação ou fortalecimento de um projeto de educação ambiental, ou diretas, com imposição de multas e limitação do uso. É importante ressaltar que, se a situação existente for melhor que a estabelecida na Etapa 6 e evidenciar uma tendência à degradação do ambiente, os indicadores e os limites máximos aceitáveis para essa área específica deverão ser revistos e readequados à realidade local.

\section{Etapa 9 - Avaliação e seleção das ações de manejo adequadas}

Dentre as ações de manejo estabelecidas na etapa anterior, seleciona-se uma ou mais alternativas, cuja relação custo/benefício esteja dentro dos objetivos e realidade local. O tempo, os recursos sociais, os materiais disponíveis e o fator financeiro são variáveis imprescindíveis na seleção e realização desse trabalho. Decidir a melhor alternativa não é uma tarefa fácil. Desse modo, o sistema LAC permite a participação do público, focalizando seus maiores interesses. Com isso, objetiva-se chegar a um consenso, garantindo que os principais problemas sejam identificados e tratados (Hendee \& Dawson, 2002).

\section{Etapa 10 - Implantação das ações e do sistema de monitoramento}

Além da implantação das ações de manejo selecionadas na Etapa 9, institui-se neste ponto um programa de monitoramento em que as condições existentes (demonstradas por um conjunto de indicadores de impacto) são comparadas com os limites máximos aceitáveis de impacto estabelecidos na Etapa 6. Além do feedback fornecido freqüentemente pelo monitoramento, é necessário verificar a performance desse sistema, ou seja, se as condições naturais e recreativas estão realmente sendo melhoradas ou mantidas nos níveis adequados. Caso contrário, as etapas 4, 6 e 9 deverão ser revistas. A dinâmica com os objetivos de manejo da área é peça fundamental para o sucesso do sistema de planejamento LAC.

\section{Seleção de indicadores de impacto}

Considerando que não é possível medir todos os componentes do ecossistema, Merigliano (1987) sugere que sejam avaliados alguns indicadores de impacto. Estes indicadores nada mais são do que variáveis específicas que, individualmente ou em combinação, são tomadas como indicativos da condição de uma área.

Os indicadores fornecem informações sobre quanto de mudança tem ocorrido, destacam os problemas e nos advertem para possíveis práticas de manejo que possam minimizá-los. Além disso, eles podem sinalizar a necessidade de ações corretivas de manejo, avaliar a eficiência de várias alternativas e ajudar a determinar se os objetivos da unidade estão sendo atingidos ou não (Merigliano, 1990).

De acordo com Leung \& Marion (2000), nos últimos quinze anos os indicadores de impacto têm recebido grande destaque na adoção e implementação de sistemas de manejo, como LAC e VERP. Analisando as principais características que 
deveriam estar presentes nos indicadores, Graefe, Kuss \& Vaske (1990) ressaltam, de forma resumida, que os indicadores mais utilizados são aqueles que podem ser observáveis de forma direta, que sejam fáceis de medir, que estejam relacionados aos objetivos de manejo da área e que sejam sensíveis às mudanças nas condiçōes de uso e passíveis de manejo.

Realizando um levantamento sobre os critérios que influenciam na seleção de indicadores de impacto, Takahashi (1998) destaca os critérios apresentados por Stankey et al. (1985) e complementado por Merigliano (1990), como segue:

a) apresentar baixo custo para coleta e análise, com um bom nível de precisão;

b) estar relacionado com a questão dos visitantes;

c) ser quantificável;

d) ser controlado pelo manejo;

e) detectar mudanças pequenas causadas pela atividade humana;

f) ser medido por pessoas não especializadas, com equipamentos e técnicas simples.

Considerando a realidade brasileira, em que predomina a escassez de recursos humanos e financeiros, a escolha adequada dos indicadores é uma etapa de fundamental importância, uma vez que permite aos administradores das unidades de conservação a otimização dos seus recursos.

Com o objetivo de avaliar quais os indicadores mais representativos para o monitoramento do uso público em duas unidades de conservação no Estado do Paraná, Takahashi (1998) realizou uma pesquisa e concluiu que os melhores indicadores ecológicos a ser monitorados na área de acampamento selvagem no Parque Estadual Pico do Marumbi (PR) foram: a macro e a microporosidade do solo, a resistência do solo à penetração nos primeiros 5 centímetros e entre 5 e 10 centímetros e a densidade do solo. Os demais indicadores recreativos avaliados (lixo espalhado, danos observados na vegetação, número de acessos secundários, número de marcas de fogueira e área total desprovida de vegetação) deveriam ser monitorados principalmente em decorrência da facilidade de obtenção, baixo custo, ligação com a atividade recreativa e possibilidade de manejo. Para a Trilha do Salto, na Reserva Particular do Patrimônio Natural Salto Morato, os principais indicadores ecológicos foram: a resistência do solo à penetração entre 5 e 10 centímetros, a macro e a microporosidade do solo e o conteúdo de carbono. Em relação aos indicadores recreativos, foi sugerido o monitoramento do lixo espalhado, o número de danos observados na vegetação, o número de acessos secundários e a largura da trilha.
Analisando os impactos decorrentes do uso recreativo na trilha RebouçasSede no Planalto do Parque Nacional do Itatiaia, Magro (1999) detectou que a declividade, a textura do solo original e a área da seção transversal são os fatores que têm maior relação com os impactos ocasionados. Segundo a pesquisadora, o grau de compactação do solo deve ser utilizado somente quando se tem o controle da visitação e quando se pretende identificar uma relação mais estreita entre o comportamento da vegetação e o aumento do uso da área.

Analisando trinta indicadores de impacto no Parque Estadual Intervales (SP), Passold (2002) concluiu que os melhores indicadores para o monitoramento do uso público foram: presença de serrapilheira, o número de árvores com raízes expostas, a área de vegetação degradada, o número de trilhas não oficiais, a presença de lixo e o número de árvores danificadas. Esse resultado deveu-se à facilidade de eles serem manejados e não apresentarem diferenças quando avaliados por diferentes indivíduos.

Ao avaliar indicadores ecológicos e recreativos em trilhas e áreas de acampamento no Parque Nacional do Itatiaia, Barros (2003) concluiu que a presença de erosão, degraus, pedras e falta de drenagem são indicadoresinerentes às condiçōes do local, tendo relação direta com a falta de planejamento e não propriamente com o comportamento inadequado dos visitantes. Indicadores como a presença de lixo e danos por vandalismo estão relacionados ao comportamento do visitante, mas não apresentaram diferenças significativas. Com isso, a autora sugere que o programa de educação do visitante privilegie técnicas de mínimo impacto relacionados a esses indicadores.

\section{Definição do limite aceitável de impacto}

Considerando que o termo indicador se refere ao impacto ou condição de uma área (Stankey et al., 1985), o limite diz respeito ao nível de impacto que é aceitável para os diferentes indicadores (Whittaker \& Shelby, 1992). Por definição, os limites envolvem valores de julgamento e, assim, devem estar profundamente embutidos nas metas e objetivos de cada unidade (Merigliano, 1987).

Para Whittaker \& Shelby (1992), as principais características desejáveis para a definição dos limites aceitáveis de impacto são:
a) ser quantificável;
b) estar relacionado com um tempo preestabelecido;
c) ser atingível;
d) focalizar o nível de impacto aceitável. 
Estes limites são estabelecidos para definir as condições que refletem o grau de naturalidade da área ou que influenciam na qualidade da experiência do visitante. Ainda, por causa do compromisso da unidade em garantir a proteção dos recursos e o acesso às oportunidades recreativas, os limites definem o compromisso que desejamos, além das condições que desejamos (Cole \& Stankey, 1997).

\section{Monitoramento dos indicadores}

De acordo com o Departamento do Interior dos EUA, monitorar os recursos naturais pode ser definido como colecionar dados sistematicamente e analisá-los em intervalos regulares, a fim de detectar as mudanças induzidas (naturalmente ou pelo ser humano) e fornecer a base para um manejo adequado.

Pesquisadores como Wagar (1964), Stankey et al. (1985); Hendee, Stankey \& Lucas (1990) e Merigliano (1987) destacam que o monitoramento deve ser realizado para assegurar e conservar os recursos naturais que fornecem valores recreativos, educativos, científicos, culturais, históricos eterapêuticos. Portanto, dependendo dos objetivos da área, há maior ou menor número de técnicas viáveis para o monitoramento do ecossistema. Um esforço pioneiro para monitorar áreas frágeis foi realizado em 1962, no Glacier Peak Wilderness, para recuperar as áreas degradadas e controlar o tipo e a intensidade de uso no lago (Hendee, Stankey \& Lucas, 1990).

O primeiro método específico publicado para monitorar sistematicamente áreas de acampamento foi desenvolvido por Hendee et al. (1976) e denominado Code-A-Site System. Em seguida, outros estudos foram desenvolvidos, embora poucos tenham sido publicados (Cole, 1989).

Como o monitoramento alerta o administrador da área em relação à tendência do indicador de impacto, os resultados do monitoramento auxiliam na avaliação da eficiência do atual programa e melhora os futuros. Se o monitoramento demonstra que as condições aceitáveis estão se deteriorando ou estão piores do que os limites estabelecidos, novas ações devem ser adicionadas (Hendee, Stankey \& Lucas, 1990).

Buttrick (1984) destaca que um programa de monitoramento deve ser implementado para atender às obrigações legais; definir as respostas às práticas de manejo adotadas; indicar ameaças reais ou potenciais; e avaliar o progresso em relação aos objetivos globais de conservação.

Merigliano (1987) estabelece dez diretrizes básicas que merecem destaque no monitoramento pelo fato de fornecerem um feedback a respeito da eficácia de certas ações de manejo, permitindo que os administradores possam planejar suas ações com maior segurança: a) definir os objetivos (por que monitorar?):

b) colecionar dados em relação às características biológicas, físicas e humanas da área, além de identificar atributos únicos;

c) identificar as atividades humanas que impactam as condições naturais;

d) selecionar os indicadores das condições ambientais e da experiência dos visitantes;

e) descrever os métodos de amostragem e medição para cada indicador;

f) treinar o pessoal de campo e implementar o monitoramento;

g) estabelecer um sistema de manejo dos dados;

h) analisar os dados coletados;

i) especificar os limites aceitáveis de câmbio e identificar ações corretivas de manejo se as condições estiverem abaixo dos padrões;

j) avaliar e redefinir métodos de medição, indicadores e padrões.

Marion \& Farrell (1998) ressaltam que o monitoramento oferece o mecanismo para avaliar periodicamente as condições dos recursos, limites ou efetividade de manejo das ações implementadas. Com isso, a incorporação de um programa de monitoramento é crucial para avaliar o sucesso das estratégias de manejo e determinar se o impacto numa área tem aumentado, diminuído ou se permanece o mesmo.

\section{Conclusão}

Os danos causados no ambiente natural resultam da combinação de fatores como a falta de planejamento, a fragilidade natural das áreas, a fiscalização ineficiente e o comportamento inadequado dos visitantes. Todavia, existem meios de detectar esses impactos para evitar que eles se agravem ou, pelo menos, minimizar seus efeitos.

Várias metodologias têm sido propostas para o planejamento e manejo do uso público em unidades de conservação, porém, a maioria delas apresenta grande similaridade e difere apenas na sequiência de etapas ou mesmo em alguns pequenos detalhes. O que ocorre na prática é que cada instituição adota o sistema que ela própria desenvolveu. Dessa forma, pode-se simplificar, destacando que qualquer método que proponha pelo menos a definição de indicadores de impactos ecológicos e recreativos, o estabelecimento de limites aceitáveis de impactos e a elaboração de um programa de monitoramento, aliado ao bom senso do administrador, oferecerá um excelente instrumento de manejo do uso público. 
Embora as experiências referentes às pesquisas e aplicação do sistema LAC no Brasil sejam limitadas a algumas áreas, ele tem sido apontado como um meio adequado para avaliar se os objetivos de manejo da unidade estão sendo alcançados ou não e quais as principais ações de manejo para atingir os objetivos preestabelecidos. Além disso, uma das principais vantagens do LAC é a possibilidade de ele ser amplamente utilizado em outras áreas, principalmente quando se trata de planejar a capacidade de carga recreativa de um local, onde o conflito em questão é estabelecer um compromisso entre a conservação e a utilização dos recursos.

\section{Referências bibliográficas}

BARROS, M. I. A. 2003. 121 p. Caracterização da visitação, dos visitantes e avaliação dos impactos ecológicos e recreativos do Planalto do Parque Nacional do Itatiaia. Dissertação (Mestrado) Escola Superior de Agricultura Luiz de Queiroz/usP, Piracicaba.

BORRIE, W. T., McOOL, S. F. \& STANKEY, G. H. 1998. Protected areas planning: principles and strategies. In: LINDBERG, K., WOOD, M. E. \& ENGELDRUM, D. Ecotourism: a guide for planners and managers. North Bennington: The Ecotourism Society, v. 2. p. 133-154.

BUTTRICK, S. C. 1984. Biological monitoring: the nature conservancy's perspective. Ogden, UT: General Technical Report IN1. usuA Forest Service, n. 173. p. 1-80.

CIFUENTES, M. 1992. Determinación de capacidad de carga turística en áreas protegidas. Turrialba: Centro Agronómico Tropical de Investigación Y Enseñanza - CATIE.

COLE, D. 1989. Low-impact recreational practices for wilderness and backcountry. Ogden, UT: General Technical Report INT. USDA Forest Service, n. 265. p. 1-131.

COLE, D. N. \& McCOOL, S. F. 1997. Limits of acceptable change and related planning process: progress and future directions. Proceedings. May 20-22, 1997. Ogden, UT: General Technical Report - INT. USDA Forest Service n. 371.71 p

COLE, D. N. \& STANKEY, G. H. 1997. Historical development of limits of acceptable change: conceptual clarifications and possible extensions. Ogden, UT: General Technical Report INT. USDA Forest Service, n. 371, p. 5-20.

DRUMM, A. \& MOORE, A. 2003. Desenvolvimento do ecoturismo: um manual para profissionais de conservação. Virginia: The Nature Conservancy, v.1.

EAGLES, P. F., McCOOL, S. F. \& HAYNES, C. D. 2002. Sustainable tourism in protected areas: guidelines for planning and management. Gland: $1 \mathrm{UCN} /$ World Commission on Protected Areas (WCPA) - Best practice protected areas guideline series $\mathrm{n}^{\circ} \mathbf{8}$.

FENNELL, D. A. 2002. Ecoturismo: uma introduçāo. Sāo Paulo: Contexto.

FRISSELL, S. S. \& STANKEY, G. H. 1972. Wilderness environmental quality: search for social and ecological harmony. In: Proceedings of the 1972 national convention; 1972 October 1-5; Hot Springs, AR. Washington, DC: Society of American Foresters.
GRAEFE, A .R., KUSS, F. R. \& VASKE, J. J. 1990. Visitor impact management: the planning framework. Washington, DC: National Parks and Conservation Association, (S.n.t)

HAMMITT, W. E. \& COLE, D. N. 1998. Wild land recreation: ecology and management. 2. ed. New York: John Wiley \& Sons.

HENDEE, J. C. et al. 1976. Code-A-Site: a system for inventory of dispersed recreational sites in roaded areas, back country, and wilderness. Research Paper PNW. Ogden, UT: USA Forest Service, n. 209. p. 1-33.

HENDEE, J.C., STANKEY, G. H. \& LUCAS, R. C. 1990. Wilderness management. Golden: North American Press.

HENDEE, J. C. \& DAWSON, C. P. 2002. Wilderness management: stewardships and protection of resources and values. 3. ed. Ogden: Fulcrum Publishing

LEUNG, Y. F. \& MARION, J. L. 2000. Recreation impacts and management in wilderness: a state-of-knowledge review. In: WILDERNESS SCIENCE IN A TIME TO CHANGE CONFERENCE: WILDERNESS ecosystem, threats, and manacement, 5. Missoula, 2000. Proceedings. Missoula: usda, Forest Service.

MAGRO, T. C. 1999.135 p. Impactos do uso público em uma trilha do Parque Nacional do Itatiaia Tese (Doutorado) - Escola de Engenharia de São Carlos/UsP, São Carlos.

MARION, J. L. \& FARRELL, T. A. 1998. Managing ecotourism visitation in protected areas. In: LINDBERG, K., WOOD, M. E. \& ENGELDRUM, D. Ecotourism: a guide for planners and managers. North Bennington: The Ecotourism Society, v. 2. p. 155-182.

MERIGLIANO, L. 1987.273 p. The identification and evaluation of indicators to monitor wildernes conditions. Thesis. University of Idaho, College of Forestry, Wildlife and Range Sciences.

1990. Indicators to monitor the wilderness recreation experience. In: LIME, David W.

(Ed.). Managing America's enduring wilderness resource. Minneapolis: University of Minnesota. p. 156-162.

McCOOL, S. F. 1996. Limits of acceptable change: a framework for managing national protected areas: experiences from the United States. Paper presented at Workshop on Impact Management in Marine Parks, Maritime Institute of Malaysia, August 13-14, Kuala Lumpur, Malaysia. Disponível em: <http://leopold.wilderness.net>. Acesso em: 8 dez. 2004.

PASSOLD, A J. 2002.75 p. Seleção de indicadores para o monitoramento do uso públcio em áreas naturais. Dissertação (Mestrado) - Escola Superior de Agricultura Luiz de Queiroz/usp, Piracicaba.

STANKEY, G. H. et al. 1985. The limits of acceptable change (LAC:) system for wilderness planning. Ogden, uT: Gen. Techn. Rep. INT. USDA Forest Service, n. 176. 37 p.

STRASDAS, W. 2002. The ecotourism training manual for protected areas managers. Zschortau: German Foundation for International Development - DSE.

TAKAHASHI, L.Y. 1997. Limite Aceitável de Câmbio (LAC): manejando e monitorando visitan-

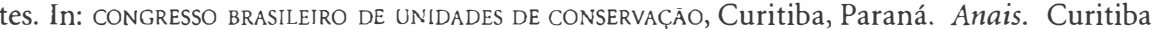
UNILIVRE. p. 445-464 
TAKAHASHI, L. Y. 1998. Caracterização dos visitantes, suas preferências e percep̧̧ões e avaliação dos impactos da visitação pública em duas unidades de conservação do Estado do Paraná. Tese (Doutorado) - Setor de Ciências Agrárias, Universidade Federal do Paraná, Curitiba.

WAGAR, J. A. 1964. The carrying capacity of wild lands for recreation. ForestScience-Monograph 7 , Washington. $24 \mathrm{p}$.

WEARING, S. \& NEIL, J. 1999. Ecoturismo: impacto, tendencias y posibilidades. Madrid: Editorial Síntesis.

WHITTAKER, D. \& SHELBY, B. 1992. Developing good standards: criteria, characteristics, and sources. Ogden, uT: General Technical Report PNw. USDA. Forest Service, n. 305. p. 6-12.

Recebido em: 10/01/2005.

Aprovado em: 20/06/2005. 